\title{
Exploration the potential mechanism of the SIRT1 and its target gene FOX01/PPARGC1A in uteropelvic junction obstruction
}

\author{
Qian Zhao, Ge Liu, Xiaoming Yin, Xu Fan, Yi Yang \\ Department of Pediatric Urology, Shengjing Hospital of China Medical University, Shenyang, China \\ Contributions: (I) Conception and design: Q Zhao, Y Yang; (II) Administrative support: Y Yang; (III) Provision of study materials or patients: Q Zhao, \\ Y Yang; (IV) Collection and assembly of data: G Liu, X Yin; (V) Data analysis and interpretation: X Fan; (VI) Manuscript writing: All authors; (VII) \\ Final approval of manuscript: All authors. \\ Correspondence to: Yi Yang. Department of Pediatric Urology, Shengjing Hospital of China Medical University, 36 Sanhao Street, Shenyang 110004, \\ China. Email: yangyilab@163.com.
}

Background: Uteropelvic junction obstruction (UPJO) is a common surgical condition, which refers to the blockage of urine flowing through kidney into proximal upper ureter. However, the underlying mechanism of UPJO is poorly understood, especially the regulated and targeted genes of sirtuin 1 in UPJO.

Methods: We sequenced three renal tissues on the obstructed side of independent children with $<20 \%$ differential renal function (DRF) and three samples with $>40 \%$ DRF. Gene expression values were obtained and compared for differentially expressed genes (DEGs). Protein-protein interaction (PPI) analysis was conducted to identify the overlapping proteins of DEGs and Sirtuin 1 (SIRT1). The co-expression genes of overlapped genes were computed using Pearson correlation coefficient. The potential role of SIRT1 gene in UPJO was explored by resequencing 3 microarray data from RNA interference (RNAi) SIRT1 lines of renal tubular epithelial (NRK52E) cells in rat and three control datasets were sequenced again. The DEGs were obtained as parallel. GO/KEGG enrichment analysis and co-expression network were conducted to explore the underlying mechanism, particularly shared pathways or function in GO/KEGG enrichment analysis results.

Results: A total of 427 up-regulated genes and 1,099 down-regulated genes were identified among 3 mRNAseq of renal tissue on the obstructed side of the independent children with $<20 \%$ DRF and 3 samples with $>40 \%$ DRF. According to prediction using the Search Tool for Retrieval of Interacting Genes/Proteins, 2 PPIs, FOXO1 and PPARGC1A, were identified among 2,524 DEGs, predicted as targets of SIRT1. Gene set enrichment analysis (GSEA) of their co-expression genes showed they may co-participate in biological activities including fatty acid degradation, regulation of signal transduction by p53 mediator. Moreover, GSEA results of DEGs was confirmed through RNAi SIRT1 lines of rat renal tubular epithelial (NRK52E) cells.

Conclusions: UPJO may cause abnormal phenotypic changes of renal tubular epithelial cells through SIRT1/FOXO1 mediated protein transport, establishment of protein localization, and intracellular transport. In addition, UPJO is involved in regulation of signal transduction, regulation of intracellular estrogen receptor signaling pathways, and nucleoprotein localization through SIRT1/PPARGC1A-mediated p53 mediators, causing abnormal phenotypic changes in renal tubular epithelial cells.

Keywords: Uteropelvic junction obstruction (UPJO); Sirtuin 1 (SIRT1); FOXO1; PPARGC1A; kidney function

Submitted Aug 06, 2021. Accepted for publication Oct 09, 2021.

doi: $10.21037 /$ tau-21-752

View this article at: https://dx.doi.org/10.21037/tau-21-752 


\section{Introduction}

Ureteropelvic junction obstruction (UPJO) is a common congenital surgical condition (1-3). It involves blockage of the flow of urine through the kidney into proximal upper ureter. The pathogenesis of UPJO can be explained by gross changes in the ureteral wall $(4,5)$. The majority of UPJO cases are congenital and caused by mechanical ureteral obstruction $(4,6)$. Timely treatment of UPJO is important to avoid chronic infection, urolithiasis, and frequent deterioration of renal function $(7,8)$. Although UPJO be addressed surgically, some patients still experience kidney disease after surgery. The damage does not recover or even progresses, and the long-term renal function deteriorates, eventually requiring hemodialysis and kidney transplantation, which seriously affects the quality of life of children and their families (9-11). Although it is a fairly common disease, there is still a lack of understanding of the underlying mechanism of UPJO. Recently, several studies have reported that many different proteins participate in regulating the formation of mechanical ureteral obstruction and leading to UPJO (12). However, in-depth research on the regulation mechanism of early renal injury in obstructive nephropathy, early diagnosis and early treatment, and delaying the renal function damage caused by obstructive nephropathy, has great economic and social significance in the familial and broader social settings.

Silent information regulator factor 2-related enzyme 1 (Sirtuin 1, SIRT1) is an important protein of the class III histone deacetylases family, which performed as a nicotinamide adenine dinucleotide-dependent deacetylase, participating in deacetylating histone proteins and other transcription factors. SIRT could also regulate many different biological processes such as gene transcription, energy metabolism, and oxidative stress (13-24). Abundantly expressed in the kidney, SIRT1 participated in renal physiologic and pathologic phenotypes. In addition to the unilateral ureteral obstruction model, the up-regulation of SIRT1 in murine renal medullary interstitial cells was reported to repress the COX2 level during oxidative stressinduced to recovery the interstitium from inflammation and fibrogenesis (25). Moreover, the SIRT1 knockout mice was showed to be impairment of angiogenesis, reduction of matrilytic activity, and retention of the profibrotic cleavage substrate tissue transglutaminase as well as endoglin-accompanied MMP-14 suppression (26-28). Recovery of MMP-14 expression in SIRT1-knock out mice was reported as many phenotypic changes including improving the angiogenic and matrilytic functions of the endothelium, inhibiting renal dysfunction, even attenuating nephrosclerosis (29). SIRT1 is a promising drug targeting for inhibiting the progression of nephrosclerosis. There have been some experiments of acute nutrient withdrawal which have mentioned that the forkhead transcription factor Foxo3a could be stimulated during interacting with $\mathrm{p} 53$ $(25,30)$. Through combining 2 p 53 binding sites at SIRT1 promoter, Foxo3a could induce the transcription of SIRT1.

However, the role and potential mechanism of the SIRT1 and its target gene FOXO1/PPARGC1A in UPJO remain unclear. We firstly investigated the regulatory role of SIRT1 and its downregulated gene expression during UPJO through RNA sequence. Therefore, we explored the expression change of genes regulated by SIRT1 in RNAi SIRT1 lines of rat renal tubular epithelial (NRK52E) cells, in which the expression of SIRT1 was knocked down. Concurrently, we compared the differential expression between messenger RNA (mRNA)-seq data of the renal tissue on the obstructed side of children with $<20 \%$ DRF and 3 samples with $>40 \%$ DRF. To further explore SIRT1, we analyzed its effect based on the prediction of proteinprotein interaction (PPI). We studied the related signaling pathway through Gene Ontology/Kyoto Encyclopedia of Genes and Genomes (GO/KEGG) enrichment analysis and conducted SIRT1-related co-expression network. In summary, the aim of our study was to examine the potential mechanism of the SIRT1 and its target gene FOXO1/ PPARGC1A in UPJO. We present the following article in accordance with the MDAR reporting checklist (available at https://dx.doi.org/10.21037/tau-21-752).

\section{Methods}

\section{Clinical data acquisition and extraction}

The mRNA-seq samples were from renal tissue on the obstructed side of the independent children with unilateral UPJO undergoing surgical treatment from Department of Pediatric Urology, Shengjing Hospital of China Medical University, the differential renal function in the treatment group was less than $20 \%$, indicating severely impairment, while it was greater than $40 \%$ in the control group, indicating that the renal function was not impaired or was slightly injured. The admission criteria for children in the treatment group was the renal dynamic scan, which indicated that their renal function was less than $20 \%$. The entry criteria for children in the control group was that 
the radionuclide examination indicated that their kidney function was greater than $40 \%$. The age of children at the time of surgery ranged from 6 to 18 weeks, or 1.5 to 5 months, and all participants were boys. Specimens of kidney tissue were acquired from the children during surgery. After the samples were collected, they were placed directly in liquid nitrogen, and then transferred to storage at $-80{ }^{\circ} \mathrm{C}$ until the time of inspection. All procedures performed in this study involving human participants were in accordance with the Declaration of Helsinki (as revised in 2013). The study was approved by institutional Ethics Review Board of Shengjing Hospital of China Medical University (No. 2013PS81K) and informed consent was taken from all the patients.

\section{Cell culture, transfection and RT-qPCR}

Normal rat kidney 52E (NRK52E) cells were purchased from American Type Culture Collection (ATCC), cultured in Dulbecco's modified Eagle medium (DMEM; Gibco, Life Technologies, Carlsbad, CA, USA) containing 10\% heat-inactivated fetal bovine serum (FBS; Corning, Corning, NY, USA) and 1\% antibiotic-antimycotic (Gibco, Life Technologies, USA).

The small interfering RNA (siRNA) of SIRT1 was transfected in the NRK52E cells using Lipofectamine 2000 (Invitrogen, Carlsbad, CA, USA) according to the manufacturer's instructions. RT-qPCR was used to detect the expression SIRT1 after transfection for $24 \mathrm{~h}$.

\section{Differentially expressed genes}

The samples were grouped based on the information of the samples, and the differential expression values of sequencing data were calculated by $\mathrm{R}$ package edge R (https://cran. r-project.org/web/packages/edgeRun/edgeRun.pdf) (31) and balltown software. Based on the cutoff of false discovery rate (FDR) value 0.05 , differentially expressed genes (DEGs) from the mRNA-seq were determined with the following threshold value: $\log 2$ | fold change $(\mathrm{FC}) \mid=1.2$, and DEGs from the microarray data were determined with the threshold value $\log 2$ I fold change $\mid=1.5$.

\section{PPI network prediction}

The online tool of Search Tool for Retrieval of Interacting Genes/Proteins (STRING) (32) was used to predict the PPI of the SIRT1 gene. At the same time, the required confidence (combined score) $>0.7$ was selected as the threshold value of PPI.

\section{Construction of co-expression network}

A co-expression network was constructed with gene pairs with Pearson correlation coefficient (PCC) values calculated by $\mathrm{R}$ of greater than 0.99 . The co-expression network is displayed by Cytoscape (https://cytoscape.org/) (33).

\section{GO/KEGG enrichment analysis}

Based on the GO (34) and KEGG pathway databases (35), the candidate genes were used to conduct GO/KEGG functional enrichment. The statistical algorithm (Fisher's exact test) was used to determine which specific functional items were most related to a group of genes. Each item in the analysis results corresponds to a statistical $\mathrm{P}$ value to represent significance. The smaller the $\mathrm{P}$ value, the greater the relationship between the item and the input genes, that is, most of the genes in the group had the function described by the term. The whole analysis procedure was showed in Figure 1.

\section{Statistical analysis}

GraphPad Prism 8 were performed for analysis. R package edgeR (https://cran.r-project.org/web/packages/edgeRun/ edgeRun.pdf) was used to calculate the differential expression values. Retrieval of Interacting Genes/Proteins (STRING) was used for PPI network prediction. Cytoscape (https://cytoscape.org/) was performed for construction of co-expression network. The mRNA expression quantification was perform using $2^{-\Delta \Delta C t}$ values. Statistical significance was defined as $\mathrm{P}<0.05$.

\section{Results}

\section{Identification of DEGs}

A total of six mRNA-seq samples were available for further analysis, including three samples from the renal tissue on the obstructed side of the independent children with less than $20 \%$ DRF and three samples from renal tissue on the obstructed side of the independent children with greater than $40 \%$ DRF. The gene expression values in these samples were obtained and compared for DEGs between samples of renal tissue on the obstructed side of the experimental 


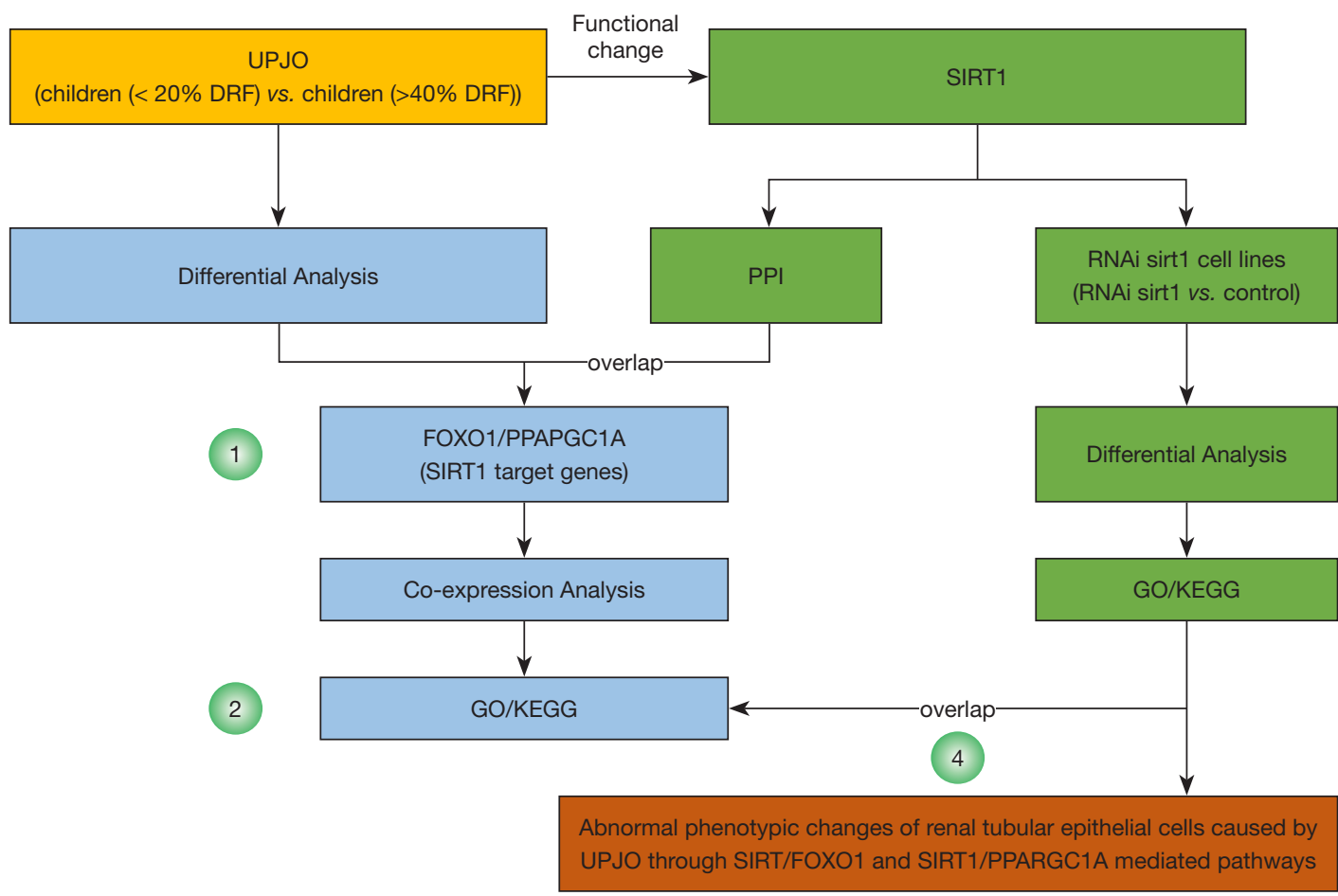

Figure 1 Flow diagram of the analysis procedure: data sources, preprocessing, analysis and validation. GO, Gene Ontology; KEGG, Kyoto Encyclopedia of Genes and Genomes; UPJO, ureteropelvic junction obstruction.

group and the three control group samples. Accordingly, 1,427 up-regulated genes and 1,099 down-regulated genes were acquired in the mRNA-seq data sets from the renal tissue on the obstructed side of the experimental group samples versus the control group samples (Figure 2A). The up-regulated genes included ADIPOQ, GSTT1, and GSTM1, among others, while the down-regulated genes were $K C N E 1 B, R G P D 2$, and $H L A-D R B 5$, among others. In addition, 3 microarray data from RNAi SIRT1 lines of rat renal tubular epithelial (NRK52E) cells and 3 control microarray data were sequenced to explore the potential role of genes regulated or targeted by SIRT1 in UPJO. Similarly, the expression levels of genes in these samples were obtained for computing the DEGs. As a result, there were 2,382 up-regulated genes and 2,138 down-regulated genes in RNAi SIRT1 lines of rat renal tubular epithelial (NRK52E) cells, respectively (Figure 2B). The up-regulated genes included Wrb, Col1a1, Fam198b, and others, while the down-regulated genes were Loxl2, Plscr1, Nucks1, and so on.

\section{Prediction of the target genes of SIRT1 in UPFO}

Firstly, the proteins that interact with SIRT1 were predicted using the online tools in STRING. From the perspective of PPI network of SIRT1 (Figure $3 A$ ), the interacting relationships covering 11 proteins were shown clearly. For example, SIRT1 protein was shown to interact with FOXO1, PPARGC1A, EP300, and so on. Among these interacted proteins, SIRT1 and HDAC1 are members of the histone deacetylases family (36). To further ensure the key target genes of SIRT1, overlap analysis was conducted on DEGs from RNA-seq and the SIRT1-interacted proteins (Figure 3B). We took all 2,526 up/down-regulated genes (DEGs) from mRNA-seq samples of the renal tissue on the obstructed side of the experimental group versus the control group samples to analyze the overlap with SIRT1-interacted proteins. Then, there were only 2 overlap genes remaining of potential importance in the pathways related with SIRT1. The 2 genes were FOXO1 $(\log 2 \mathrm{FD}=-0.30, \mathrm{P}=0.035)$ and PPARGC1A $(\log 2 \mathrm{FD}=-0.62, \mathrm{P}=0.0002)$, which may be the target genes of SIRT1 in UPJO.

\section{The prediction of pathway related to SIRT1 through the co-expression network}

Firstly, FOXO1 gene was selected for construction of its 
A

Case vs. control

· Up (1427) · Middle (15844) · Down (1099)

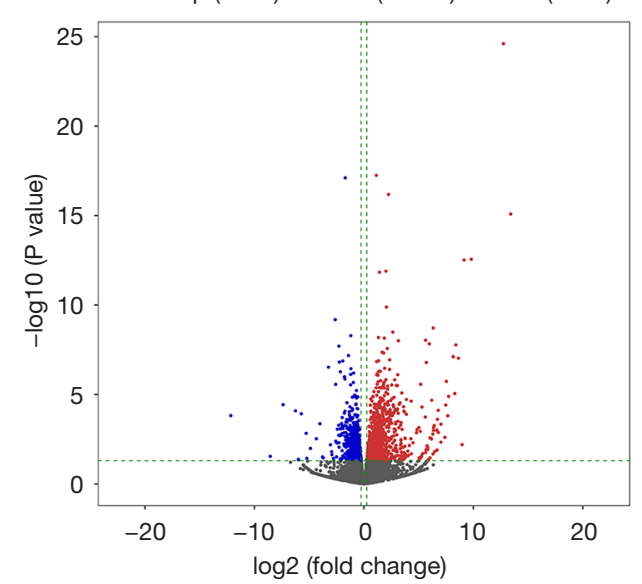

B

KD vs. NC

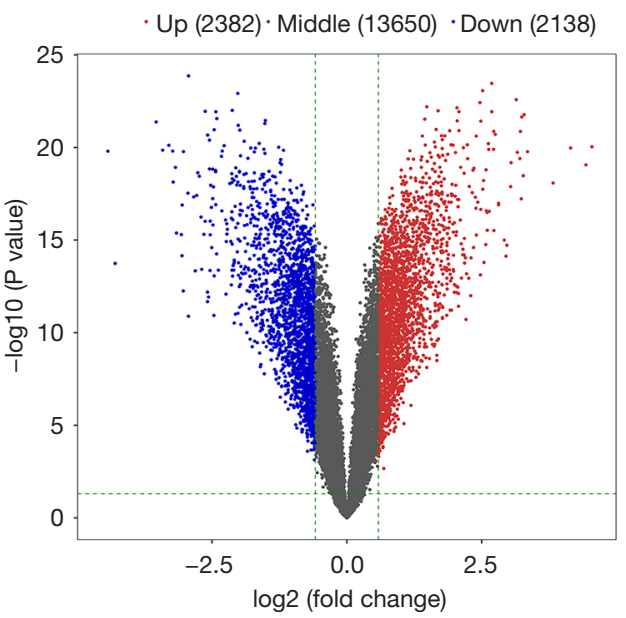

Figure 2 Volcano maps of differential expressed genes. (A) A volcano map of differential expressed genes from mRNA-seq samples of renal tissue on the obstructed side of children with less than $20 \%$ DRF versus the 3 samples with greater than $40 \%$ DRF; (B) a volcano map of differential expressed genes from RNAi SIRT1 lines of rat renal tubular epithelial (NRK52E) cells versus control samples. The $\mathrm{x}$ axis in the map is the fold change of relative expression rates between the 2 samples (with logarithmic treatment), and the y axis is the statistical test value, i.e., $\mathrm{P}$ value. The higher $-\log 10$ (P value) was, the more significant the difference was. Each dot in the graph demonstrated a gene, the red dot shows the up-regulative gene, the blue dot indicates the down-regulative gene, and the gray dot indicates no significant difference gene. DRF, differential renal function; mRNA, messenger RNA.

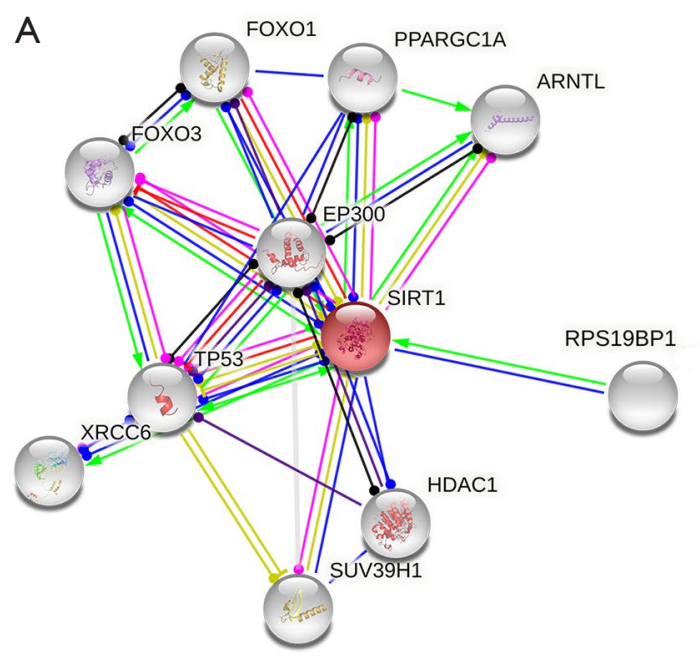

\section{B}

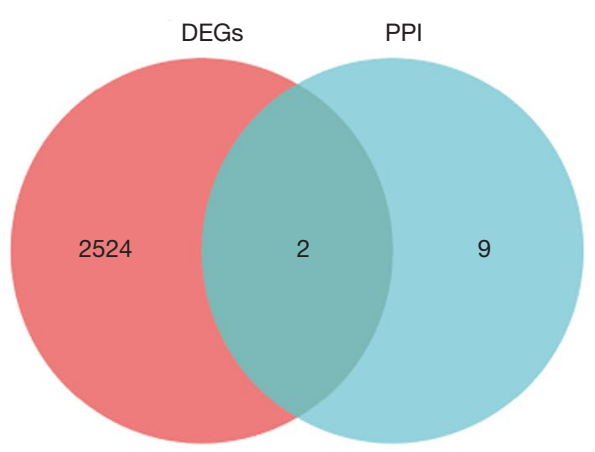

Figure 3 PPI network of SIRT1 and its Venn diagram. (A) Nodes represent genes and edges indicate interaction between 2 proteins. The more edges connected to a node, the more important the protein is in the network. (B) Venn diagram of 2 gene sets. One gene set in red represents the DEGs obtained from the mRNA-seq data of the renal tissue on the obstructed side of the independent children with $<20 \%$ DRF versus samples with $>40 \%$ DRF. The other gene set in blue indicates the proteins interacted with SIRT1 gene. PPI, protein-protein interaction; DEG, differentially expressed gene; DRF, differential renal function. 
co-expression networks, respectively. Firstly, co-expression FOXO1 and its GSEA were mainly identified based on their intersecting PCC values, the results showed the co-expression genes of FOXO1 were shown to include ZC3H15, ZZZ3, ANO10, INVS, and UBE2G1 (Figure 4A). We conducted enrichment analysis of the down-regulated genes from mRNA-seq samples of the renal tissue on the obstructed side of the experimental group due to the downregulation gene FOXO1. Positive expression with FOXO1 was detected in these genes. Through GO enrichment analysis of FOXO1, the co-expression genes were shown to be mainly enriched in the process of carboxylic acid metabolism, organic acid metabolism, monocarboxylic acid metabolism, carboxylic acid decomposition, and other biological processes (BP; Figures 4B). In KEGG pathways, they mainly involved in metabolic pathways, fatty acid degradation, and the degradation of valine, leucine, and isoleucine (Figure 4C). Moreover, the heatmap of GO enrichment analysis results (Figure $5 A$ ) and the heatmap of GO enrichment analysis results with the fold change of gene expression (Figure $5 B$ ) were also proved that the co-expression genes were enriched in those biological processes.

Then, co-expression PPARGC1A and their GSEA were mainly demonstrated dependent on their intersecting PCC values (Figure $6 A$ ), and the co-expression genes of PPARGC1A included GOLIM4, CHORDC1, ZNF470, $S L T M, K I F 16 B$, and so forth. The co-expression genes of PPARGC1A were subjected to GO (Figure 6B) enrichment analysis. The co-expression genes of PPARGC1A were mainly enriched in the GO terms of protein localization to nucleolus, regulation of signal transduction by class $\mathrm{p} 53$ mediator, monocarboxylic acid metabolism, carboxylic acid decomposition, and other BP (Figures $6 B, 7 A, 7 B$ ), while there were no significant enriched terms in the analysis of KEGG pathway for them.

\section{Further validation of pathways related to SIRT1 in RNAi SIRT1 lines of rat renal tubular epithelial (NRK52E) cells}

To further validate the pathways related to SITR1, we conducted the GO enrichment analysis of up-regulated genes in RNAi SIRT1 lines of rat renal tubular epithelial (NRK52E) cells versus control samples. After RNAi SIRT1 lines of rat renal tubular epithelial (NRK52E) cells, RTqPCR was performed to confirm that the expression of SIRT1 in SIRT1-RNAi NRK52E cells was significantly downregulated (Figure S1). However, there is not significant phenotype change of NRK52E cells before and after RNAi
Sirt transfection (photos were not showed). The analysis results suggested that these genes are mainly enriched in $\mathrm{BP}$ such as positive regulation of protein metabolism, tissue development, regulation of protein metabolism process, and regulation of signal transduction (Figure $8 A$ ). The KEGG pathway enrichment analysis revealed that they were likely involved in cancer associated proteoglycan, AGE-RAGE signaling pathway, FOXO signaling pathway, endocrine resistance, and other signaling pathways (Figure 8B).

Next, we performed Venn diagram analysis of the above GO terms and FOXO1/PPARGC1A co-expression gene enrichment results (Figure $8 C, 8 D$ ). On the one hand, it was revealed that FOXO1 co-expressed gene enrichment results and RNAi sirt1 cell lines' enrichment results shared 85 GO BP terms (Table 1), mainly including protein transport, establishment of protein localization, intracellular transport, regulation of ubiquitin-dependent protein catabolism, and other biological processes. Accordingly, UPJO may cause abnormal phenotypic changes of renal tubular epithelial cells through SIRT1/FOXO1 mediated protein transport, the establishment of protein localization, and intracellular transport. On the other hand, it was shown that PPARGC1A co-expression gene enrichment results and RNAi SIRT1 cell lines' enrichment results shared 21 GO BP terms (Table 2), including p53-type mediators to regulate signal transduction, the regulation of intracellular estrogen receptor signaling pathways, nuclear protein localization and other BP. Overall, is was determined that UPJO regulates signal transduction, regulation of intracellular estrogen receptor signaling pathways, and nucleoprotein localization through SIRT1/PPARGC1A-mediated p53 mediators, causing abnormal phenotypic changes in renal tubular epithelial cells.

\section{Discussion}

The disease UPJO is characterized by the blockage during urine flowing through the kidney into proximal upper ureter. The pathogenesis of UPJO is related to gross changes in the ureteral wall $(4,5)$. Therefore, we analyzed samples from the renal tissues of children with DRF $<20 \%$ and $>40 \%$. There were 427 up-regulated genes and 1,099 down-regulated genes obtained from the datasets from the renal tissues of children with DRF $<20 \%$ versus with samples with DRF $>40 \%$. Differential analysis was also performed in the RNAi SIRT1 cell lines and control cell lines, which indicated the expression changes of genes might be regulated or targeted by SIRT1 in the UPJO-related 
A

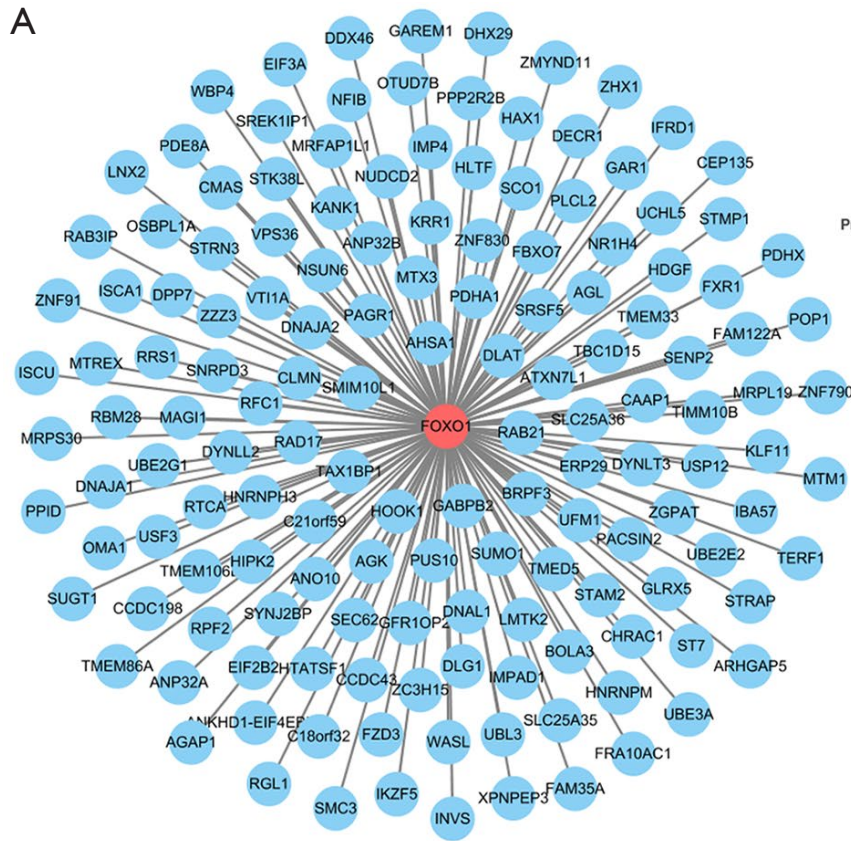

C

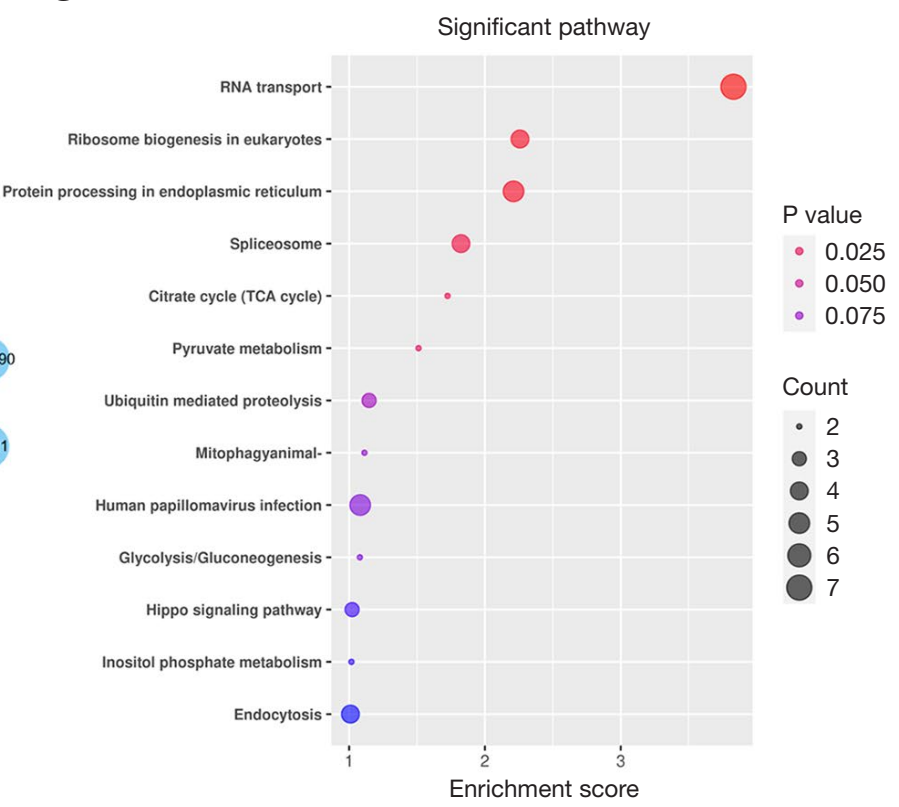

Significant go terms

\section{B}

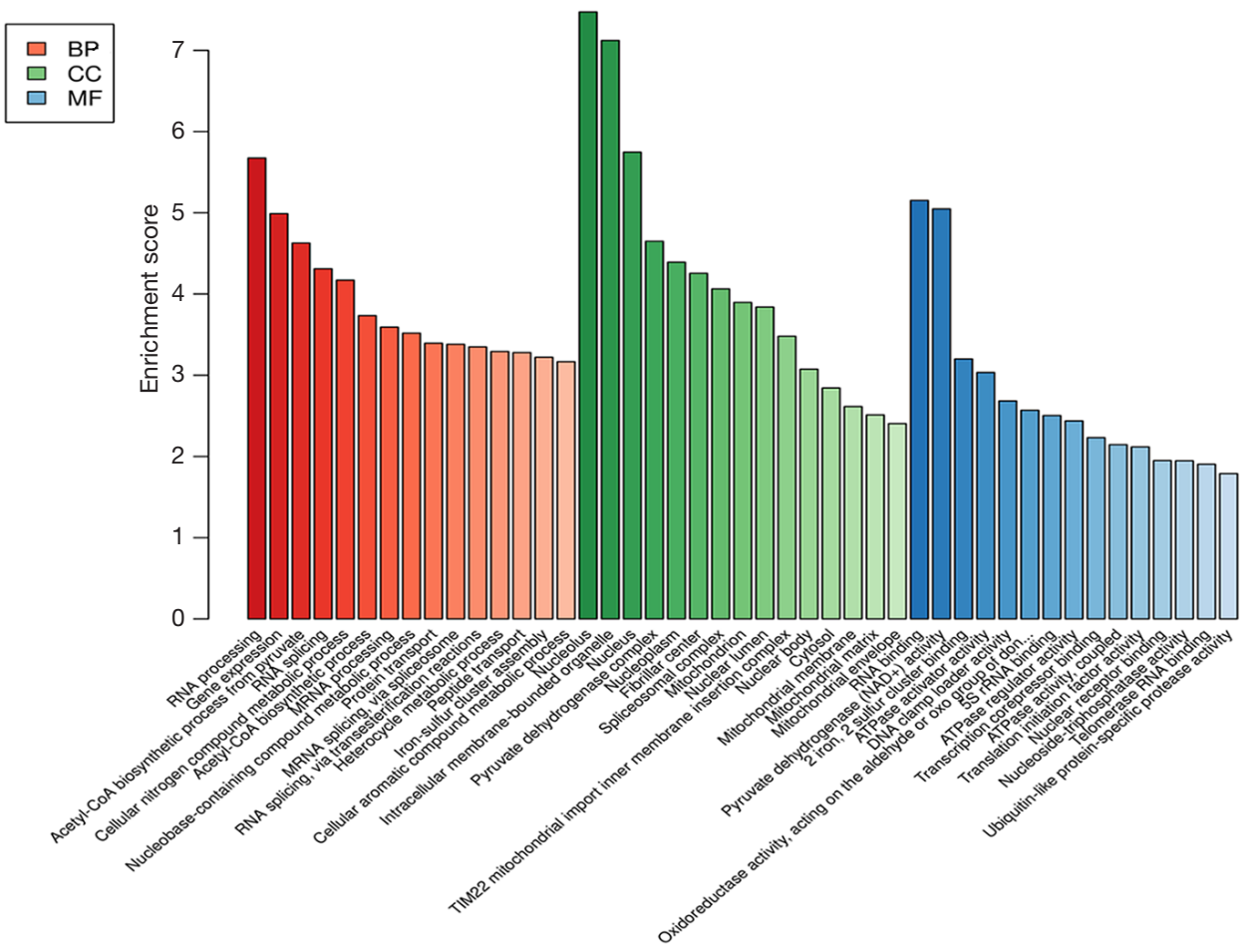

Figure 4 The co-expression network of gene FOXO1 and their GSEA. (A) The co-expression network of gene FOXO1. (B) GO enrichment analysis of the co-expression genes of FOXO1. The $\mathrm{x}$ axis demonstrated the GO term, and the y axis show the enrichment level, corresponding to the height of the column. The greater the enrichment score value, the more significant the GO term is enriched. The 3 colors represent 3 categories: biological process (BP), cellular component (CC) and molecular function (MF). (C) KEGG pathway enrichment analysis of the co-expression genes of FOXO1. The vertical axis showed the KEGG pathway name, and the horizontal axis represents enrichment score. The larger the value, the greater the enrichment degree. The size represents the genes' number in the pathway, and the color of the point represents the different P value. GSEA, gene set enrichment analysis; GO, Gene Ontology; KEGG, Kyoto Encyclopedia of Genes and Genomes. 

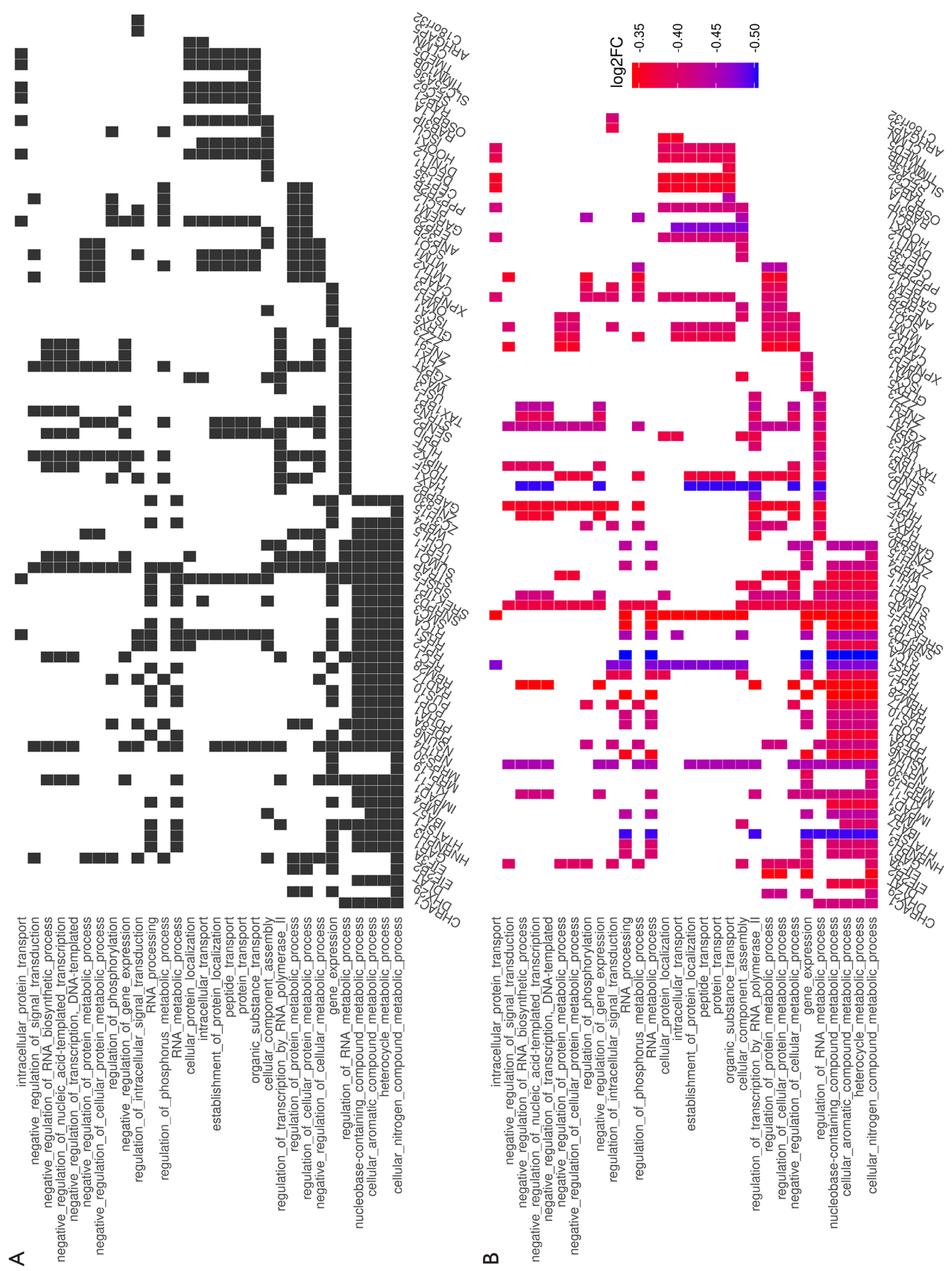

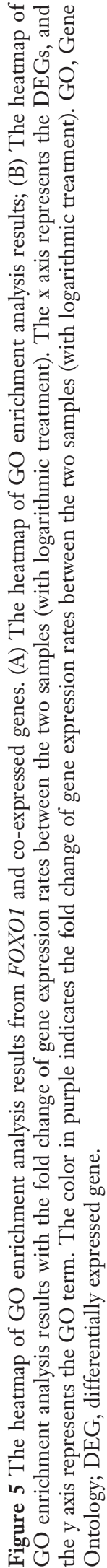

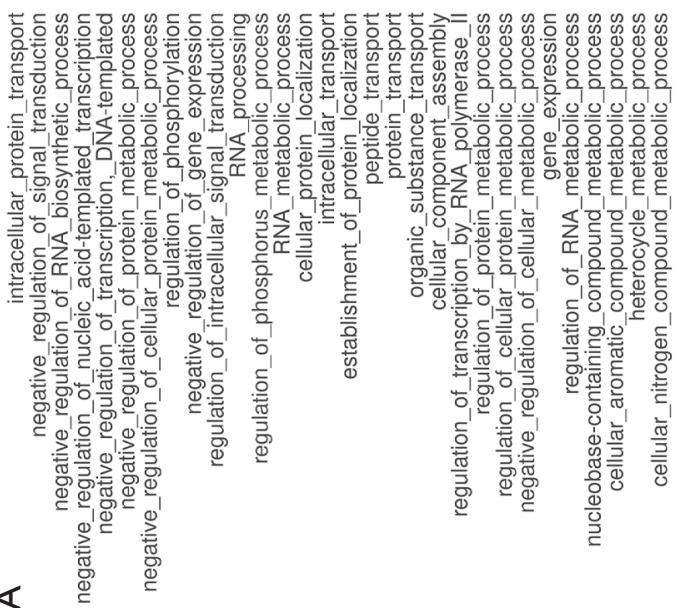


A

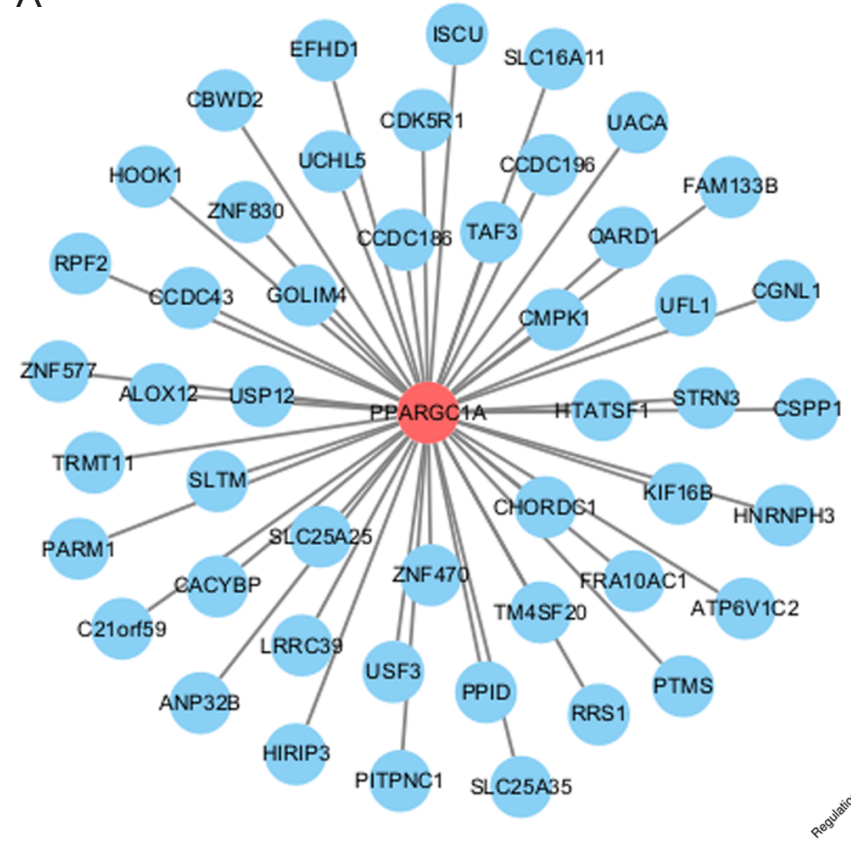

B

Significant go terms

Figure 6 The co-expression network of PPARGC1A and their GSEA analysis. (A) The co-expression network of PPARGC1A. (B) GO enrichment analysis of the co-expression genes of $P P A R G C 1 A$. The $\mathrm{x}$ axis represents the GO term, and the $\mathrm{y}$ axis represents the significance level of enrichment, corresponding to the height of the column. The greater the enrichment score value, the more significantly the GO term is enriched. GSEA, gene set enrichment analysis; GO, Gene Ontology.

pathways and BP caused by the knock down of SIRT1.

We predicted the PPIs of SIRT1 using the STRING database, one of which was HDAC1. This relationship corresponds with the previous published views of the function of SIRT1 and HDAC1 (36). Catalytic-independent neuroprotection by SIRT1 is mediated through interaction with HDAC1. To elucidate their role in UPJO, follow-up studies will need to be conducted in the future. As all 2,524 DEGs only had 2 interacting proteins predicted, FOXO1 and PPARGC1A were identified as serving as the targets of SIRT1. SIRT was reported to deacetylate and activate the PPARGC1A and FOXO1 to effect gluconeogenesis in liver (37). Moreover, as reported by Cheng et al. (38), FOXO1 could inhibit the acetylation of PPARGC1A to recover mitochondrial oxidation. Moreover, FOXO1 as transcription factors was reported to be coactivated by PPARGC1A to regulate the downstream protein expression including gluconeogenic target proteins (39). Moreover, both FOXO1 and FOXO3 as transcription factors likely combine 2 p5 3 binding sites in the SIRT1 promoter, and stimulate SIRT1 transcription, but FOXO3 is outside the scope of DEGs from the mRNA-seq datasets of children.

Through the co-expression network analysis, it was revealed that their co-expression genes seem to act with them in UPJO. The GSEA analysis of FOXO1 coexpression genes clearly specified that they may take part in biological activity together, including fatty acid degradation, and the degradation of valine, leucine, and isoleucine. Similarly, the GSEA analysis of PPARGC1A co-expression genes implicitly reinforced that they have a relationship with protein localization to nucleolus, regulation of signal transduction by class p53 mediator, monocarboxylic acid metabolism, carboxylic acid decomposition, and other BP.

At the moment, there have been 2,382 up-regulated genes and 2,138 down-regulated genes identified in RNAi SIRT1 cell lines, respectively. The GSEA results were subjected to overlap analysis with the above terms of coexpression genes from the target genes of gene SIRT1. Based upon the entire summarized analysis, UPJO may give rise to abnormal phenotypic changes of renal tubular epithelial cells through SIRT1/FOXO1 protein localized and mediated intracellular protein transport. Lastly, UPJO 


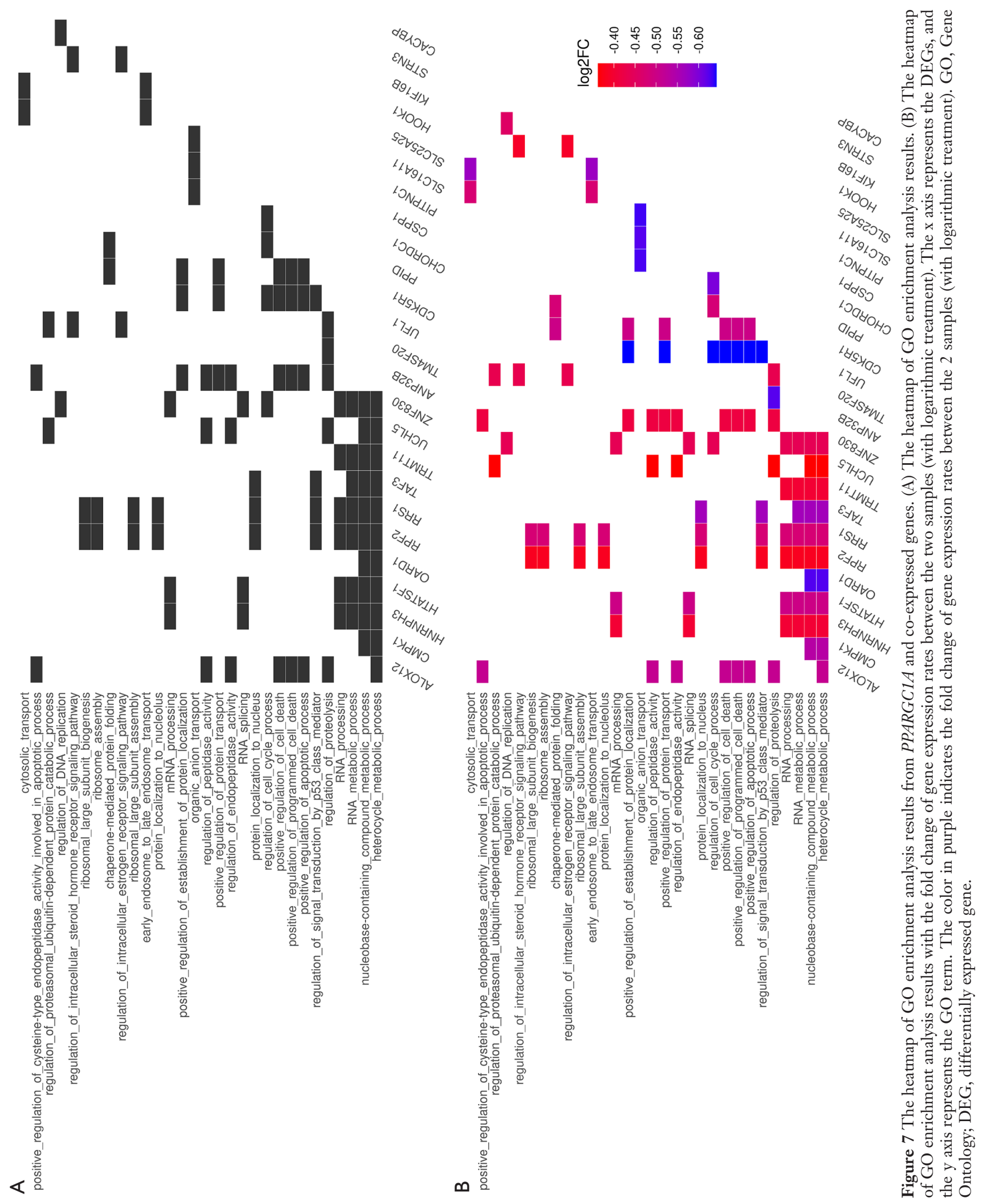


A Significant go terms

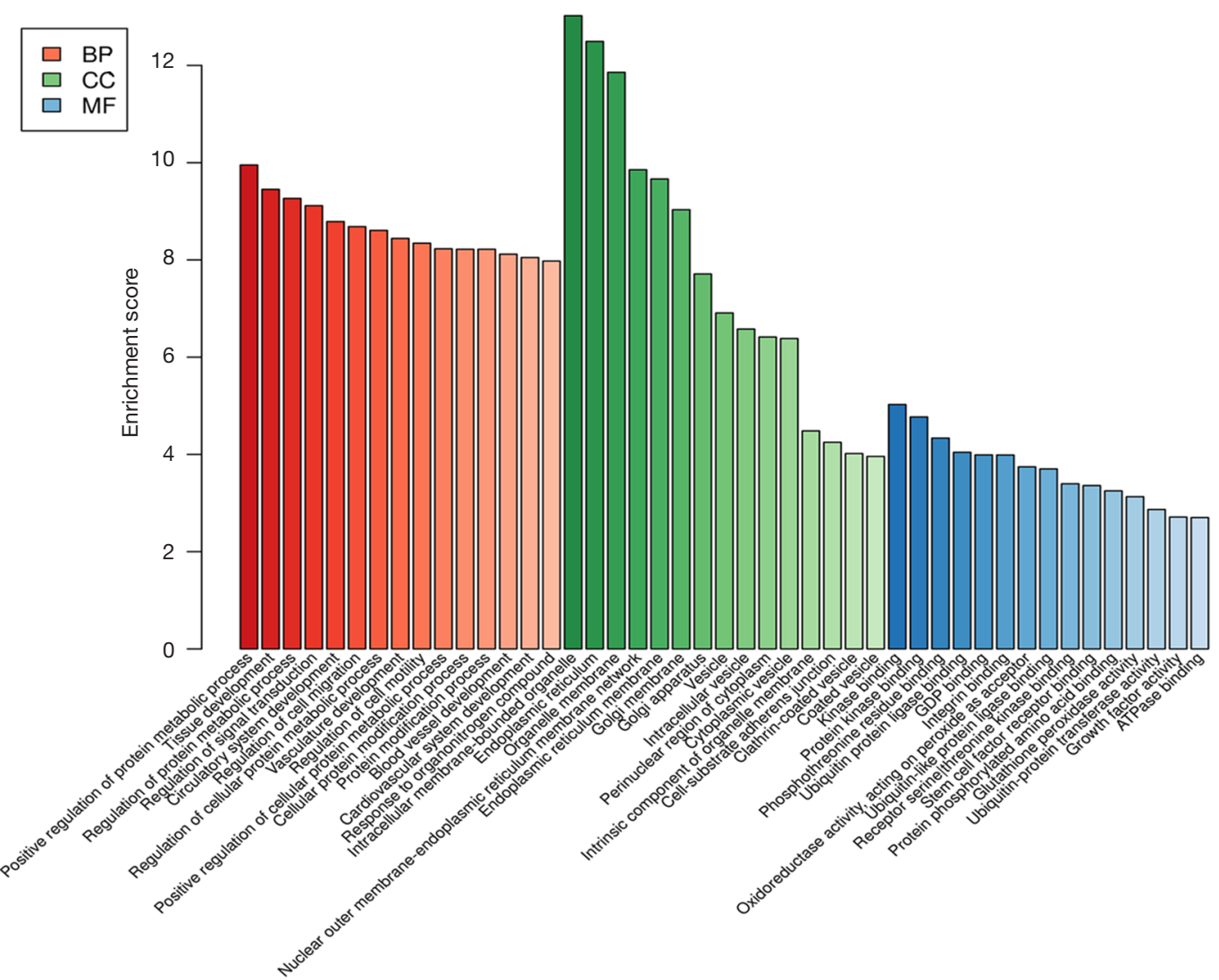

B

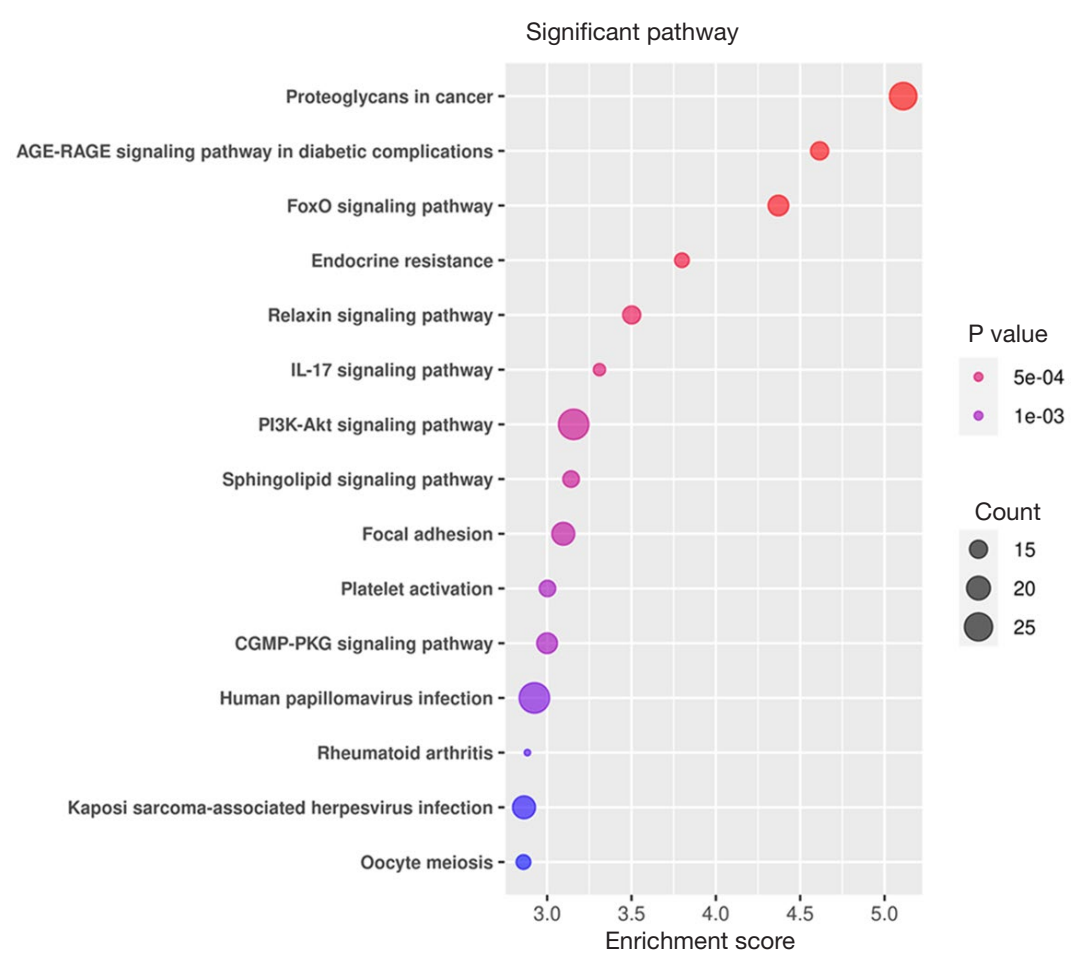



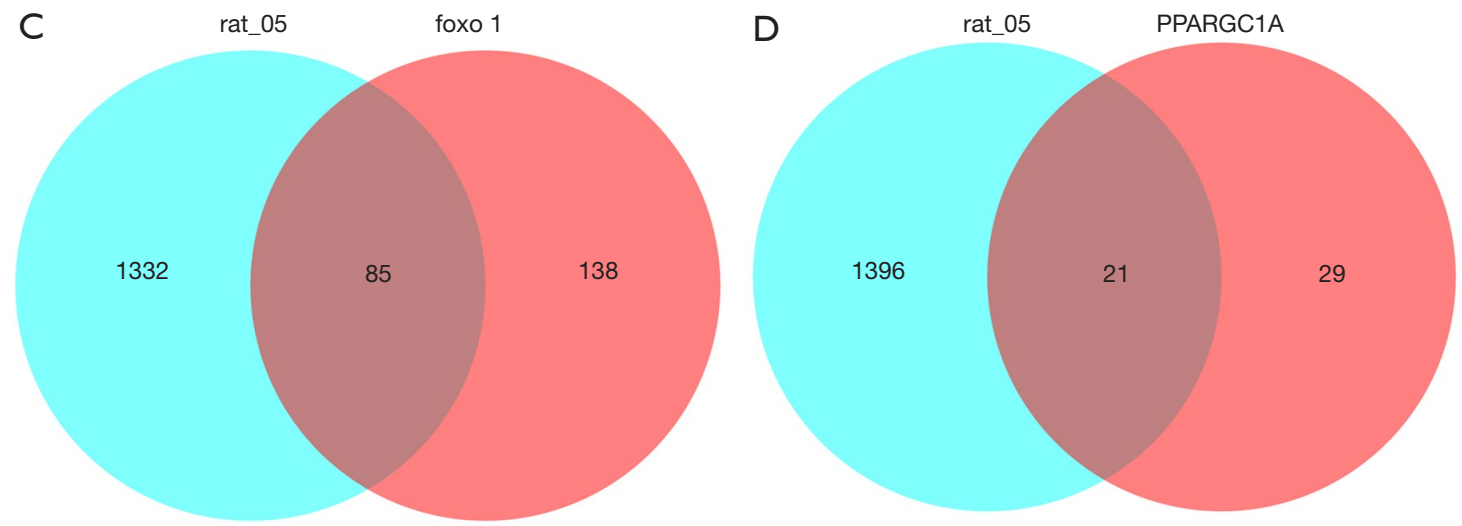

Figure 8 Analysis of up-regulated genes in RNAi SIRT1 lines versus control lines. (A) GO enrichment analysis of the up-regulated genes in RNAi SIRT1 lines. The $\mathrm{x}$ axis represents the GO term, and the $\mathrm{y}$ axis represents the significance level of enrichment, corresponding to the height of the column. The greater the enrichment score value, the more significant the GO term is enriched. (B) KEGG pathway enrichment analysis of the upregulated genes in RNAi sirt1 lines. The vertical axis represents the KEGG pathway name, and the horizontal axis represents enrichment score. The larger the value, the greater the enrichment degree. The size of the point indicates the number of genes in the pathway, and the color of the point corresponds to different $\mathrm{P}$ value ranges. (C) Venn diagram of 2 GO term gene sets. One gene set in blue represents the all GO terms (BP, CC, MF) obtained from the enrichment analysis of the up-regulated genes in RNAi sirt1 lines. The other gene set in red indicates all GO terms (BP, CC, MF) from enrichment analysis of the co-expression genes of FOXO1. (D) Venn diagram of two GO term gene sets. One gene set in blue represents the all GO terms (BP, CC, MF) obtained from the enrichment analysis of the up-regulated genes in RNAi sirt1 lines. The other gene set in red indicates all GO terms (BP, CC, MF) from enrichment analysis of the co-expression genes of PPARGC1A. The circles with different colors show the GO terms of different sample groups, the values represent the common or unique GO terms among different groups, the sum of all numbers in the circle showed the total number of GO terms in the group, and the cross area of the circle represents the total number of GO terms among the groups. GO, Gene Ontology; KEGG, Kyoto Encyclopedia of Genes and Genomes; BP, biological process; CC, cellular component; MF, molecular function.

Table 1 Partial shared BP terms from the enrichment analysis of the up-regulated genes in RNAi SIRT1 cell lines and the coexpression genes of PPARGC1A

\begin{tabular}{ll}
\hline ID & Term \\
\hline GO:0015031 & protein_transport \\
GO:0015833 & peptide_transport \\
GO:0045184 & establishment_of_protein_localization \\
GO:0046907 & intracellular_transport \\
GO:2000058 & regulation_of_ubiquitin-dependent_protein_ \\
& catabolic_process \\
\hline
\end{tabular}

BP, biological process; GO, Gene Ontology.

is involved in SIRT1/PPARGC1A-mediated p53 regulation of signal transduction and intracellular estrogen receptor signaling pathways on the account of nucleoprotein localization, and leads to abnormal phenotypic changes in renal tubular epithelial cells. However, there is some limitation that we did not identify the directly regulation effect among SIRT1, FOXO1 and PPARGC1A.
Table 2 Partial shared BP term from the enrichment analysis of the up-regulated genes in RNAi sirt1 cell lines and the co-expression genes of FOXO1

\begin{tabular}{ll}
\hline ID & Term \\
\hline GO:1901796 & $\begin{array}{l}\text { regulation_of_signal_transduction_by_p53_ } \\
\text { class_mediator }\end{array}$ \\
GO:0033146 & $\begin{array}{l}\text { regulation_of_intracellular_estrogen_ } \\
\text { receptor_signaling_pathway }\end{array}$ \\
GO:0034504 & protein_localization_to_nucleus \\
GO:0033143 & $\begin{array}{l}\text { regulation_of_intracellular_steroid_hormone_ } \\
\text { receptor_signaling_pathway }\end{array}$ \\
GO:0030162 & regulation_of_proteolysis
\end{tabular}

$\mathrm{BP}$, biological process; GO, Gene Ontology.

\section{Conclusions}

We concluded that UPJO may cause abnormal phenotypic changes of renal tubular epithelial cells through SIRT1/ FOXO1 mediated protein transport, the establishment of protein localization, and intracellular transport. In 
the meantime, UPJO is involved in regulation of signal transduction, regulation of intracellular estrogen receptor signaling pathways, and nucleoprotein localization through SIRT1/PPARGC1A-mediated p53 mediators, causing abnormal phenotypic changes in renal tubular epithelial cells.

\section{Acknowledgments}

Funding: National Natural Science Foundation of China (grant No. 81571514), 345 talent project and Key Research and Develop Program Collabrative Planning Project of Liaoning Province (2020JH 2/10300145)

\section{Footnote}

Reporting Checklist: The authors have completed the MDAR reporting checklist (available at https://dx.doi.org/10.21037/ tau-21-752).

Data Sharing Statement: Available at https://dx.doi. org/10.21037/tau-21-752

Conflicts of Interest: All authors have completed the ICMJE uniform disclosure form (available at https://dx.doi. org/10.21037/tau-21-752). The authors have no conflicts of interest to declare.

Ethical Statement: The authors are accountable for all aspects of the work in ensuring that questions related to the accuracy or integrity of any part of the work are appropriately investigated and resolved. All procedures performed in this study involving human participants were in accordance with the Declaration of Helsinki (as revised in 2013). The study was approved by institutional Ethics Review Board of Shengjing Hospital of China Medical University (No.: 2013PS81K) and informed consent was taken from all the patients.

Open Access Statement: This is an Open Access article distributed in accordance with the Creative Commons Attribution-NonCommercial-NoDerivs 4.0 International License (CC BY-NC-ND 4.0), which permits the noncommercial replication and distribution of the article with the strict proviso that no changes or edits are made and the original work is properly cited (including links to both the formal publication through the relevant DOI and the license). See: https://creativecommons.org/licenses/by-nc-nd/4.0/.

\section{References}

1. Cui X, He YB, Huang WH, et al. Mini-laparoscopic pyeloplasty to treat UPJO in infants. Minim Invasive Ther Allied Technol 2020. [Epub ahead of print].

2. Zhang P, Shi T, Fam X, et al. Robotic-assisted laparoscopic pyeloplasty as management for recurrent ureteropelvic junction obstruction: a comparison study with primary pyelo-plasty. Transl Androl Urol 2020;9:1278-85.

3. Tabari AK, Atqiaee K, Mohajerzadeh L, et al. Early pyeloplasty versus conservative management of severe ureteropelvic junction obstruction in asymptomatic infants. J Pediatr Surg 2020;55:1936-40.

4. Krajewski W, Wojciechowska J, Dembowski J, et al. Hydronephrosis in the course of ureteropelvic junction obstruction: An underestimated problem? Current opinions on the pathogenesis, diagnosis and treatment. Adv Clin Exp Med 2017;26:857-64.

5. Longpre M, Nguan A, Macneily AE, et al. Prediction of the outcome of antenatally diagnosed hydronephrosis: a multivariable analysis. J Pediatr Urol 2012;8:135-9.

6. Vandervoort K, Lasky S, Sethna C, et al. Hydronephrosis in infants and children: natural history and risk factors for persistence in children followed by a medical service. Clin Med Pediatr 2009;3:63-70.

7. Carlström M. Hydronephrosis and risk of later development of hypertension. Acta Paediatr 2019;108:50-7.

8. Kandur Y, Salan A, Guler AG, et al. Diuretic renography in hydronephrosis: a retrospective single-center study. Int Urol Nephrol 2018;50:1199-204.

9. Chaabane W, Praddaude F, Buleon M, et al. Renal functional decline and glomerulotubular injury are arrested but not restored by release of unilateral ureteral obstruction (UUO). Am J Physiol Renal Physiol 2013;304:F432-9.

10. Chevalier RL, Forbes MS, Galarreta CI, et al. Responses of proximal tubular cells to injury in congenital renal disease: fight or flight. Pediatr Nephrol 2014;29:537-41.

11. Liu BC, Tang TT, Lv LL, et al. Renal tubule injury: a driving force toward chronic kidney disease. Kidney Int 2018;93:568-79.

12. Wu B, Gong X, Kennedy WA, et al. Identification of transcripts associated with renal damage due to ureteral obstruction as candidate urinary biomarkers. Am J Physiol Renal Physiol 2018;315:F16-26.

13. Alves-Fernandes DK, Jasiulionis MG. The Role of SIRT1 on DNA Damage Response and Epigenetic Alterations in 
Cancer. Int J Mol Sci 2019;20:3153.

14. Farghali H, Kemelo MK, Canová NK. SIRT1 Modulators in Experimentally Induced Liver Injury. Oxid Med Cell Longev 2019;2019:8765954.

15. Lu G, Li J, Zhang H, et al. Role and Possible Mechanisms of Sirt1 in Depression. Oxid Med Cell Longev 2018;2018:8596903.

16. Meng X, Tan J, Li M, et al. Sirt1: Role Under the Condition of Ischemia/Hypoxia. Cell Mol Neurobiol 2017;37:17-28.

17. Han $Y$, Sun $W$, Ren D, et al. SIRT1 agonism modulates cardiac NLRP3 inflammasome through pyruvate dehydrogenase during ischemia and reperfusion. Redox Biol 2020;34:101538.

18. Iside C, Scafuro M, Nebbioso A, et al. SIRT1 Activation by Natural Phytochemicals: An Overview. Front Pharmacol 2020;11:1225.

19. Li T, Garcia-Gomez A, Morante-Palacios O, et al. SIRT1/2 orchestrate acquisition of DNA methylation and loss of histone $\mathrm{H} 3$ activating marks to prevent premature activation of inflammatory genes in macrophages. Nucleic Acids Res 2020;48:665-81.

20. Nagappan A, Kim JH, Jung DY, et al. Cryptotanshinone from the Salvia miltiorrhiza Bunge Attenuates EthanolInduced Liver Injury by Activation of AMPK/SIRT1 and Nrf2 Signaling Pathways. Int J Mol Sci 2019;21:265.

21. Rada P, Pardo V, Mobasher MA, et al. SIRT1 Controls Acetaminophen Hepatotoxicity by Modulating Inflammation and Oxidative Stress. Antioxid Redox Signal 2018;28:1187-208.

22. Strycharz J, Rygielska Z, Swiderska E, et al. SIRT1 as a Therapeutic Target in Diabetic Complications. Curr Med Chem 2018;25:1002-35.

23. Zhang Y, Anoopkumar-Dukie S, Arora D, et al. Review of the anti-inflammatory effect of SIRT1 and SIRT2 modulators on neurodegenerative diseases. Eur J Pharmacol 2020;867:172847.

24. Zhao S, Li W, Cheng F, et al. High-pressure carbon dioxide pneumoperitoneum induces oxidative stress and mitochondria-associated apoptotic pathway in rabbit kidneys with severe hydronephrosis. Int J Mol Med 2019;43:305-15.

25. Nemoto S, Fergusson MM, Finkel T. Nutrient availability regulates SIRT1 through a forkhead-dependent pathway. Science 2004;306:2105-8.

26. Jiang Y, Botchway BOA, Hu Z, et al. Overexpression of SIRT1 Inhibits Corticosterone-Induced Autophagy. Neuroscience 2019;411:11-22.

27. Mokhberian N, Hashemi SM, Jajarmi V, et al. Sirt1 antisense transcript is down-regulated in human tumors. Mol Biol Rep 2019;46:2299-305.

28. Zhang D, Sun X, Chen X, et al. Ultrasound evaluation for prediction of outcomes and surgical decision in fetal hydronephrosis. Exp Ther Med 2019;18:1399-406.

29. Vasko R, Xavier S, Chen J, et al. Endothelial sirtuin 1 deficiency perpetrates nephrosclerosis through downregulation of matrix metalloproteinase-14: relevance to fibrosis of vascular senescence. J Am Soc Nephrol 2014;25:276-91.

30. Ma F, Wu J, Jiang Z, et al. P53/NRF2 mediates SIRT1's protective effect on diabetic nephropathy. Biochim Biophys Acta Mol Cell Res 2019;1866:1272-81.

31. McCarthy DJ, Chen Y, Smyth GK. Differential expression analysis of multifactor RNA-Seq experiments with respect to biological variation. Nucleic Acids Res 2012;40:4288-97.

32. von Mering C, Huynen M, Jaeggi D, et al. STRING: a database of predicted functional associations between proteins. Nucleic Acids Res 2003;31:258-61.

33. Shannon P, Markiel A, Ozier O, et al. Cytoscape: a software environment for integrated models of biomolecular interaction networks. Genome Res 2003;13:2498-504.

34. Ashburner M, Ball CA, Blake JA, et al. Gene ontology: tool for the unification of biology. The Gene Ontology Consortium. Nat Genet 2000;25:25-9.

35. Kanehisa M, Goto S. KEGG: kyoto encyclopedia of genes and genomes. Nucleic Acids Res 2000;28:27-30.

36. Pfister JA, Ma C, D'Mello SR. Catalytic-independent neuroprotection by SIRT1 is mediated through interaction with HDAC1. PLoS One 2019;14:e0215208.

37. Imai S, Guarente L. Ten years of NAD-dependent SIR2 family deacetylases: implications for metabolic diseases. Trends Pharmacol Sci 2010;31:212-20.

38. Cheng Z, Guo S, Copps K, et al. Foxo1 integrates insulin signaling with mitochondrial function in the liver. Nat Med 2009;15:1307-11.

39. Charos AE, Reed BD, Raha D, et al. A highly integrated and complex PPARGC1A transcription factor binding network in HepG2 cells. Genome Res 2012;22:1668-79.

(English Language Editor: J. Jones)

Cite this article as: Zhao Q, Liu G, Yin X, Fan X, Yang Y. Exploration the potential mechanism of the SIRT1 and its target gene FOXO1/ PPARGC1A in uteropelvic junction obstruction. Transl Androl Urol 2021;10(11):4192-4205. doi: $10.21037 /$ tau-21-752 


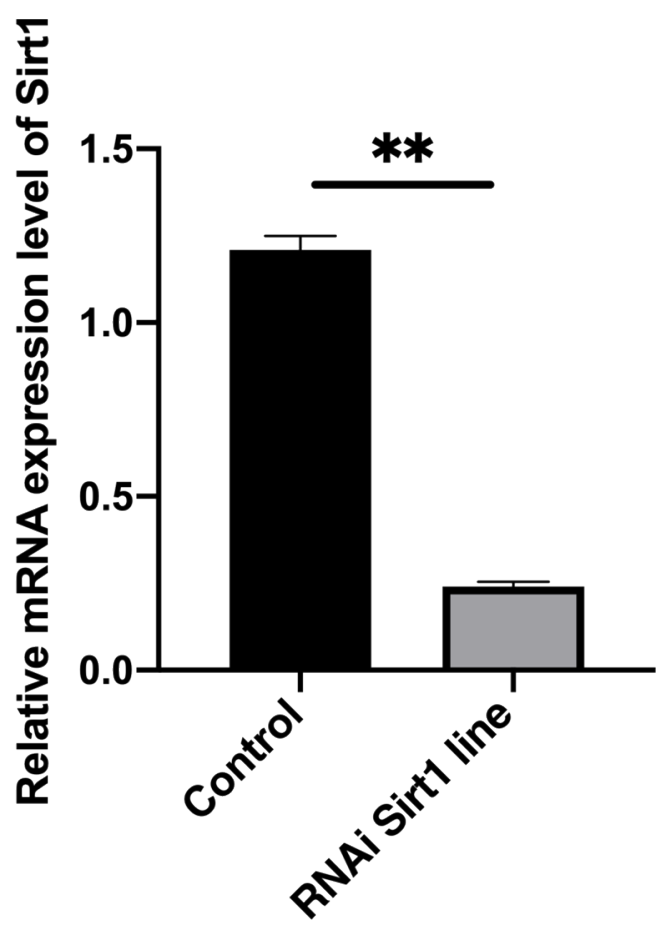

Figure S1 The RT-qPCR results of Sirt1 expression level $\left({ }^{* *} \mathrm{P}<0.01\right)$. 